\title{
MAD SUBALGEBRAS AND LIE SUBALGEBRAS OF AN ENVELOPING ALGEBRA
}

\author{
XIN TANG
}

\author{
(Received 18 March 2009)
}

\begin{abstract}
Let $\mathcal{U}(\mathfrak{r}(1))$ denote the enveloping algebra of the two-dimensional nonabelian Lie algebra $\mathfrak{r}(1)$ over a base field $\mathbb{K}$. We study the maximal abelian ad-nilpotent (mad) associative subalgebras and finite-dimensional Lie subalgebras of $\mathcal{U}(\mathfrak{r}(1))$. We first prove that the set of noncentral elements of $\mathcal{U}(\mathfrak{r}(1))$ admits the Dixmier partition, $\mathcal{U}(\mathfrak{r}(1))-\mathbb{K}=\bigcup_{i=1}^{5} \Delta_{i}$, and establish characterization theorems for elements in $\Delta_{i}$, $i=1,3,4$. Then we determine the elements in $\Delta_{i}, i=1,3$, and describe the eigenvalues for the inner derivation $\operatorname{ad}_{B} x, x \in \Delta_{i}, i=3,4$. We also derive other useful results for elements in $\Delta_{i}, i=2,3,4,5$. As an application, we find all framed mad subalgebras of $\mathcal{U}(\mathfrak{r}(1))$ and determine all finite-dimensional nonabelian Lie algebras that can be realized as Lie subalgebras of $\mathcal{U}(\mathfrak{r}(1))$. We also study the realizations of the Lie algebra $\mathfrak{r}(1)$ in $\mathcal{U}(\mathfrak{r}(1))$ in detail.
\end{abstract}

2000 Mathematics subject classification: primary 17A36, 17B35; secondary 17B60.

Keywords and phrases: Dixmier partition, mad subalgebras, Lie subalgebras.

\section{Introduction}

Let $\mathbb{K}$ be an algebraically closed field of characteristic zero. Let $A_{1}$ be the first Weyl algebra over $\mathbb{K}$, which is the $\mathbb{K}$-algebra generated by generators $p, q$ subject to the commutator relation $p q-q p=1$. Note that $A_{1}$ is a primitive quotient of the enveloping algebra of the three-dimensional Heisenberg Lie algebra. Early motivations for the study of $A_{1}$ and its higher degree analogs $A_{n}$ were the applications in physics, especially in quantum mechanics [18]. Nevertheless, the study of Weyl algebras is closely related to the algebraic study of systems of linear differential equations [7, 8, 14] and representation theory of Lie algebras [5].

The systematic study of $A_{1}$ was first initiated by Dixmier in a seminal work [9] and was continued in [10]. In [9], Dixmier proved that all noncentral elements of $A_{1}$ can be partitioned into five disjoint subsets: $\Delta_{i}, i=1,2,3,4,5$. This partition is now called the Dixmier partition. Dixmier studied the generalized eigenspace $F(x)$ of the linear operator $\operatorname{ad}_{A_{1}} x$ and determined the generators of the automorphism group $\operatorname{Aut}\left(A_{1}\right)$ of $A_{1}$. Following Dixmier, the elements in $\Delta_{1}$ (respectively in $\Delta_{3}$ ) are called strictly nilpotent elements (respectively strictly semisimple elements). These elements were

This research is supported in part by Fayetteville State University Faculty Development Research Grant. (c) 2010 Australian Mathematical Publishing Association Inc. 0004-9727/2010 \$16.00 
further explicitly classified by Dixmier up to the action of $\operatorname{Aut}\left(A_{1}\right)$ in [9]. Furthermore, for any strictly semisimple element $x \in A_{1}-\mathbb{K}$, Dixmier proved that the eigenvalues of the operator $\operatorname{ad}_{A_{1}} x$ are exactly the set $\mathbb{Z} \rho$ for some $\rho \in \mathbb{K}$. In particular, at the end of [9], Dixmier listed six problems concerning the first Weyl algebra $A_{1}$, which have stimulated further research. In [13], further important results were obtained by Joseph for elements in $\Delta_{2}, \Delta_{4}$. Dixmier's philosophy has been further studied by Bavula in [2-4]. The maximal abelian ad-nilpotent (mad) associative subalgebras of $A_{1}$ were also determined by Dixmier in [9]. In [6], Berest and Wilson generalized Dixmier's result about mad algebras to algebras of differential operators over curves, which are domains Morita equivalent to the first Weyl algebra $A_{1}$.

A natural important question is to further determine all finite-dimensional nonabelian Lie subalgebras which can be realized as Lie subalgebras of $A_{1}$. It is easy to see that the Lie algebras $\mathfrak{s l}(2), \mathfrak{s l}(2) \times \mathbb{K}$ and $\mathfrak{s l}(2) \ltimes \mathcal{H}_{3}$ (where $\mathcal{H}_{3}$ denotes the three-dimensional Heisenberg Lie algebra) can be realized as Lie subalgebras of $A_{1}$. In [11], Igusa proved a necessary condition for two elements of $A_{1}$ to generate an infinite-dimensional Lie subalgebra. In [16], Simoni and Zaccaria proved that $\mathfrak{s l}(2)$ is the only semisimple Lie algebra that can be realized in $A_{1}$. In [12], Joseph established a remarkable property of the realizations of $\mathfrak{s l}(2)$ in $A_{1}$. In [15], Rausch de Traubenberg et al. determined all finite-dimensional nonabelian Lie algebras that can be realized as Lie subalgebras of $A_{1}$ and studied the action of $\operatorname{Aut}\left(A_{1}\right) \times \operatorname{Aut}(\mathfrak{s l}(2))$ on a particular family of realizations of $\mathfrak{s l}(2)$ in $A_{1}$.

Unfortunately, a direct generalization of these results to Weyl algebras $A_{n}, n \geq 2$, is not available. However, it is still of interest and possible to address this question for other algebras closely related to $A_{1}$. The main aim of this paper is to study the mad associative subalgebras and finite-dimensional Lie subalgebras of $\mathcal{U}(\mathfrak{r}(1))$, which denotes the enveloping algebra of the two-dimensional nonabelian Lie algebra $\mathfrak{r}(1)$ according to the notation in [15]. As we will see, the enveloping algebra $\mathcal{U}(\mathfrak{r}(1))$ is indeed closely related to $A_{1}$. Let $B$ denote the Borel subalgebra of the first Weyl algebra $A_{1}$ generated by elements $p, p q$. Then it is easy to see that $B$ is isomorphic to the enveloping algebra $\mathcal{U}(\mathfrak{r}(1))$. From now on, we will not distinguish $B$ from $\mathcal{U}(\mathfrak{r}(1))$. Note that the determination of mad associative subalgebras and finite-dimensional Lie subalgebras of $B$ will be based on a detailed study of the Dixmier partition of $B-\mathbb{K}$.

To proceed, we will first show that noncentral elements of $B$ can also be partitioned into five disjoint subsets $\Delta_{i}, i=1, \ldots, 5$, according to the behavior of the generalized eigenspaces of their associated inner derivations $\operatorname{ad}_{B} x$. Then we will further analyze the generalized eigenspaces of these associated inner derivations in more detail and prove some useful results. In particular, we will establish an important characterization of these elements $x$ such that $F(x)=B$, which immediately leads to a new and elementary determination of the automorphism group $\operatorname{Aut}(B)$ of $B$. Note that the automorphism group $\operatorname{Aut}(B)$ was first determined by Smith in [17] using a different method; a simpler proof can be found in [1].

Using the above characterization, we give an explicit description of the strictly nilpotent elements of $B$ (elements in $\Delta_{1}$ ) and the strictly semisimple elements of $B$ 
(elements in $\Delta_{3}$ ). Thanks to the simple nature of the group $\operatorname{Aut}(B)$, we are able to completely determine the elements in $\Delta_{i}, i=1,3$, and describe the set of all eigenvalues of $\operatorname{ad}_{B} x$ for any $x \in \Delta_{3}$. As an application, we determine the structure of all mad subalgebras of $B$ and their framings. We also study the action of $\operatorname{Aut}(B)$ on the set of all framed mad associative subalgebras of $B$. We get a similar picture as in $[6,9]$. In addition, by presenting some concrete examples, we prove that the $\Delta_{i}$ $(i=2,4,5)$ are nonempty.

Pertaining to the determination of elements in $\Delta_{4}$, we are also able to provide a useful reduction theorem. Using this reduction theorem, we are able to prove that the eigenvalues of $\operatorname{ad}_{B} x, x \in \Delta_{4}$, are included in the set $\mathbb{Z}_{\geq 0} \rho$ for some $\rho \in \mathbb{K}$. It is very tempting to claim that this inclusion is indeed equality. However, we do not know how to prove such a claim. For elements $x \in \Delta_{3} \cup \Delta_{4}$, we prove that $C(x)=\mathbb{K}[x]$, which further implies that $f(x) \in \Delta_{5}$ for any $x \in \Delta_{3} \cup \Delta_{4}$ and any polynomial $f(X)$ with $\operatorname{deg} f>1$. In addition, we prove that $f(x) \in \Delta_{1}$ (respectively $\Delta_{2}$ ) if and only if $f(x) \in \Delta_{1}$ (respectively $\Delta_{2}$ ) whenever $f(X)$ is a nontrivial polynomial. As an application, we prove that $\mathbb{K}[x]-\mathbb{K} \subset \Delta_{5}$ for any $x \in \Delta_{5}$.

Finally, to tie up all the ends, we determine all finite-dimensional nonabelian Lie algebras which can be realized as Lie subalgebras of $B$. Indeed, we show that these Lie algebras are exactly finite-dimensional nonnilpotent solvable Lie algebras of the specific type $\mathfrak{r}\left(i_{1}, \ldots, i_{n}\right)$ as defined in [15]. This determination is mainly based on the results about the inner derivations $\operatorname{ad}_{B} x$ established in the first part of this paper and the corresponding results for Lie subalgebras of $A_{1}$ as obtained in [15]. It is easy to see that the two-dimensional nonabelian Lie algebra $\mathfrak{r}(1)$ itself can be realized as a Lie subalgebra of $B$. Thus we will further study the realizations of $\mathfrak{r}(1)$ in $B$ in detail. In particular, we give a partition of the set $B^{\mathfrak{r}(1)}$ of all realizations of $\mathfrak{r}(1)$ in $B$ as $B^{\mathfrak{r}(1)}=B_{1}^{\mathfrak{r}(1)} \cup B_{2}^{\mathfrak{r}(1)}$. We further prove that $B_{1}^{\mathfrak{r}(1)}$ is the union of orbits of a family $\mathcal{N}$ of realizations of $\mathfrak{r}(1)$ in $B$ under the action of $\operatorname{Aut}(B)$. We also construct some examples of elements in $B_{2}^{\mathfrak{r}(1)}$. In what follows, unless otherwise stated, we will always fix $\mathbb{K}$ as an algebraically closed field of characteristic zero.

The paper is organized as follows. In Section 2 we study the Dixmier partition of noncentral elements in $B$ and obtain various properties on the inner derivations $\operatorname{ad}_{B} x$. In Section 3 we characterize all strictly nilpotent elements and strictly semisimple elements in $B$; we determine the mad subalgebras of $B$ and their framings. In Section 4 we further derive some useful results on elements in $\Delta_{i}, i=2,3,4,5$. In Section 5 we determine all finite-dimensional nonabelian Lie algebras that can be realized as Lie subalgebras of $B$ and construct some specific examples of Lie subalgebras of $B$; some of these might be infinite-dimensional. In Section 6 we study the realizations of the Lie algebra $\mathfrak{r}(1)$ in $B$.

\section{Dixmier partition of elements in $B-\mathbb{K}$}

In this section, we prove that noncentral elements of $B$ can be partitioned into five disjoint sets. We give a characterization of these elements $x$ satisfying $F(x)=B$, 
where $F(x)$ is the generalized eigenspace associated with the inner derivation $\operatorname{ad}_{B} x$. As a first application, we give a new determination of the automorphism group of $B$.

2.1. A partition of elements in $\boldsymbol{B}-\mathbb{K}$. Note that the first Weyl algebra $A_{1}$ is the $\mathbb{K}$-algebra generated by $p, q$ subject to the relation $p q-q p=1$ and $B$ is the Borel subalgebra of $A_{1}$ generated by $p, p q$. It is easy to show that the center of $B$ is also reduced to the base field $\mathbb{K}$. In this subsection, we give a partition of the noncentral elements of $B$ according to the properties of the generalized eigenspaces of the associated inner derivations $\operatorname{ad}_{B} x, x \in B$.

First of all, we recall some background and notation from [9] and we refer the reader to [9] for more details. Let $A$ be any associative algebra over any base field $k$. For any $x \in A$, we denote by $\operatorname{ad}_{A} x$, or simply by ad $x$, the linear operator on $A$ defined by $\operatorname{ad}_{A} x(a)=x a-a x$ for any $a \in A$. For any $x \in A, \lambda \in k$, we denote by $F(x, \lambda, A)$, or simply by $F(x, \lambda)$, the set of all elements $y \in A$ such that $\left(\operatorname{ad}_{A} x-\lambda\right)^{n}(y)=0$ for sufficiently large $n$. We denote by $F(x, A)=\bigoplus_{\lambda \in k} F(x, \lambda)$, or simply by $F(x)$, the direct sum of all such $F(x, \lambda, A)$. It is obvious that $F(x)$ is a subalgebra of $A$ graded by $F(x, \lambda), \lambda \in k$. Denote by $N(x, A)$, or simply by $N(x)$, the set of all elements $y \in A$ such that $\left(\operatorname{ad}_{A} x\right)^{n}(y)=0$ for sufficiently large $n$. It is easy to verify that $N(x)$ is also a subalgebra of $A$, which is a filtered algebra filtered by $N(x, n), n \in \mathbb{N}$. Let $D(x, \lambda, A)$ be the set of all elements $y \in A$ such that $\operatorname{ad}_{A} x(y)=\lambda y$, and set $D(x, A)=\bigoplus_{\lambda \in k} D(x, \lambda, A)$; we denote this simply as $D(x)$. Then $D(x)$ is a subalgebra of $A$, which is graded by $D(x, \lambda), \lambda \in k$.

Let $C(x, A)$, or simply $C(x)$, denote the centralizer of the element $x$ in $A$. We state following simple lemma.

LEMMA 2.1. For any $x \in B$, we have $N(x) \cap D(x)=C(x)$.

PROOF. The proof is a direct verification and we omit it.

LEMMA 2.2. Let $\lambda \in \mathbb{K}$ and $z \in B$ such that $\left(\operatorname{ad}_{A} x-\lambda\right)^{2} z=0$. Then

$$
\left(\operatorname{ad}_{A} x-n \lambda\right)^{n} z^{n}=n !\left(\left(\operatorname{ad}_{A} x-\lambda\right) z\right)^{n}, \quad n=1,2,3, \ldots
$$

and

$$
\left(\operatorname{ad}_{A} x-n \lambda\right)^{n+1} z^{n}=0 .
$$

Proof. Since $B$ is a subalgebra of $A_{1}$, then $z$ is also an element of $A_{1}$ and the linear operator $\operatorname{ad}_{B} x$ on $B$ can be extended to a linear operator $\operatorname{ad}_{A_{1}} x$ on $A_{1}$. Thus, [9, Proof of Lemma 6.4] can be adopted here without modification, and we will not reproduce it here.

For the algebra $B$, we have the following result.

\section{LEMMA 2.3. B is a domain and has GK-dimension two.}

PRoOf. Note that $B$ can be regarded as the universal enveloping algebra of the twodimensional nonabelian Lie algebra. Thus the result follows from the PoincaréBirkhoff-Witt theorem for the universal enveloping algebras of Lie algebras. 
In addition, we have the following result for $B$, which is an analog of Proposition 6.5 for $A_{1}$ in [9].

Proposition 2.4. For any $\lambda \in \mathbb{K}^{*}$ and any $a \in B$, we have $D(a, \lambda, B)=$ $F(a, \lambda, B)$.

PRoOf. Note that [9, Proof of Proposition 6.5] depends only on the fact that $A_{1}$ is a domain and has GK-dimension two. Thanks to Lemma 2.3, we know that $B$ is a domain and has GK-dimension two. Thus we can copy [9, Proof of Proposition 6.5] word for word and we will not reproduce it here.

As a result, we also have the following corollary.

Corollary 2.5. For any $x \in B$, either $F(x)=D(x)$ or $F(x)=N(x)$.

PROOF. This follows directly from Proposition 2.4 and Lemma 2.2.

Note that the center of $B$ is reduced to the base field $\mathbb{K}$, so the set of noncentral elements in $B$ is the set $B-\mathbb{K}$. The following result gives a partition of $B-\mathbb{K}$ into five disjoint subsets.

THEOREM 2.6. The set $B-\mathbb{K}$ is the disjoint union of the following subsets:

(1) $\Delta_{1}$, the set of all elements $x \in B-\mathbb{K}$ such that $N(x)=B, D(x)=C(x)$;

(2) $\Delta_{2}$, the set of all elements $x \in B-\mathbb{K}$ such that $N(x) \neq B, N(x) \neq$ $C(x), D(x)=C(x)$;

(3) $\Delta_{3}$, the set of all elements $x \in B-\mathbb{K}$ such that $D(x)=B, N(x)=C(x)$;

(4) $\Delta_{4}$, the set of all elements $x \in B-\mathbb{K}$ such that $D(x) \neq B, D(x) \neq$ $C(x), N(x)=C(x)$;

(5) $\Delta_{5}$, the set of all elements $x \in B-\mathbb{K}$ such that $D(x)=N(x)=C(x)$.

PROOF. It is obvious that $\Delta_{i}, i=1, \ldots, 5$, are disjoint subsets of $B-\mathbb{K}$. If $x \notin \Delta_{5}$, then either $N(x) \neq C(x)$ or $D(x) \neq C(x)$. Note that $F(x)=D(x)$ or $F(x)=N(x)$, so $D(x) \cap N(x)=F(x) \cap N(x)=N(x)$ or $D(x) \cap N(x)=D(x) \cap F(x)=D(x)$. Thus $C(x)=D(x)$ or $C(x)=N(x)$. If $N(x) \neq C(x)$, then $D(x)=C(x)$. Thus $x \in \Delta_{1} \cup \Delta_{2}$. If $D(x) \neq C(x)$, then $N(x)=C(x)$. Thus $x \in \Delta_{3} \cup \Delta_{4}$. We have proved the theorem.

REMARK 2.7. Following Dixmier [9], we call the elements in $\Delta_{1} \cup \Delta_{2}$ (respectively $\Delta_{1}$ ) nilpotent (respectively strictly nilpotent), elements in $\Delta_{3} \cup \Delta_{4}$ (respectively $\Delta_{3}$ ) semisimple (respectively strictly semisimple) and elements in $\Delta_{5}$ generic. We will call this partition the Dixmier partition.

2.2. A characterization of elements $\boldsymbol{x}$ satisfying $\boldsymbol{F}(\boldsymbol{x})=\boldsymbol{B}$. In this subsection we further study the generalized eigenspaces $F(x)$ of the associated linear operator $\operatorname{ad}_{B} x$. We give a useful characterization of elements $x$ such that $F(x)=B$. As an immediate application, we give a new and elementary determination of the generators of the automorphism group $\operatorname{Aut}(B)$ of $B$. 
For any $a \in \mathbb{K}^{*}$, any $\lambda \in \mathbb{K}$ and any integer $n \geq 0$, let $\Phi_{a, n, \lambda}$ be an automorphism of $B$ defined by

$$
\Phi_{a, n, \lambda}(p)=a p, \quad \Phi_{a, n, \lambda}(p q)=p q+\lambda p^{n} .
$$

Indeed, it is easy to verify that these maps can be extended to automorphisms of $B$. When $a=1$, these automorphisms can be regarded as the restrictions of the corresponding automorphisms of $A_{1}$. We denote by $G$ the subgroup of $\operatorname{Aut}(B)$ generated by all such automorphisms $\Phi_{a, n, \lambda}$ of $B$; we shall prove that $G=\operatorname{Aut}(B)$ at the end of this subsection.

First of all, we recall some notation and technical lemmas about polynomials from [9, Section 1]. Let $f=\sum \alpha_{i, j} X^{i} Y^{j} \in \mathbb{K}[X, Y]$ be a polynomial in $X, Y$. We denote by $E(f)$ the set of integer couples $(i, j)$ such that $\alpha_{i, j} \neq 0$. Let $\rho, \sigma$ be any two real numbers. We set

$$
v_{\rho, \sigma}(f)=\sup _{(i, j) \in E(f)}(\rho i+\sigma j) .
$$

As a convention, we set $v_{\rho, \sigma}(0)=-\infty$. We denote by $E(f, \rho, \sigma)$ the set of integer couples $(i, j)$ in $E(f)$ such that $\rho i+\sigma j=v_{\rho, \sigma}(f)$. If $f \neq 0$, then $E(f, \rho, \sigma)$ is not empty. If $E(f)=E(f, \rho, \sigma)$, then $f$ is called $(\rho, \sigma)$ homogeneous with $(\rho, \sigma)$-degree $v_{\rho, \sigma}(f)$. Let $x=\sum \alpha_{i, j} p^{i} q^{j}$. In a similar fashion, we can define $E(x), v_{\rho, \sigma}(x), E(x, \rho, \sigma)$. Correspondingly, the polynomial $\sum_{(i, j) \in E(x, \rho, \sigma)} \alpha_{i, j} X^{i} Y^{j}$ is called the $(\rho, \sigma)$-associated polynomial of $x$.

LEMMA 2.8 [9, Lemma 1.3]. Let $f \in \mathbb{K}[X, Y]$ be a $(\rho, \sigma)$-homogeneous polynomial of $(\rho, \sigma)$-degree $v$. Then:

(1) $\rho X(\partial f / \partial X)+\sigma Y(\partial f / \partial Y)=v f$;

(2) if $\rho$ and $\sigma$ are linearly independent over $\mathbb{Q}$, then $f$ is a monomial.

LeMma 2.9 [9, Lemma 2.7]. Suppose $x, y$ are any two nonzero elements of $A_{1}$ and $\rho, \sigma$ are any two real numbers such that $\rho+\sigma>0$. Let us set $v=v_{\rho, \sigma}(x), \omega=$ $v_{\rho, \sigma}(y)$. And let $f_{1}, g_{1}$ be the $(\rho, \sigma)$-associated polynomials of $x, y$, respectively. Then we have the following.

(1) There exists a unique pair $(t, u)$ of elements in $A_{1}$ satisfying the following properties:

(a) $[x, y]=t+u$;

(b) $E(t)=E(t, \rho, \sigma)$ and $v_{\rho, \sigma}(t)=v+\omega-(\rho+\sigma)$;

(c) $v_{\rho, \sigma}(u)<v+\omega-(\rho+\sigma)$.

(2) In addition, the following conditions are equivalent:

(a) $t=0$;

(b) $\partial_{X}\left(f_{1}\right) \partial_{Y}\left(g_{1}\right)-\partial_{Y}\left(f_{1}\right) \partial_{X}\left(g_{1}\right)=0$.

If $v, \omega$ are integers, then these conditions are further equivalent to the condition that $g_{1}^{v}$ is proportional to $f_{1}^{\omega}$. 
(3) If $t \neq 0$, then the $(\rho, \sigma)$-associated polynomial of $[x, y]$ is just $\partial_{X}\left(f_{1}\right) \partial_{Y}\left(g_{1}\right)-$ $\partial_{Y}\left(f_{1}\right) \partial_{X}\left(g_{1}\right)$.

We now prove an important lemma concerning the generalized eigenspace of the inner derivation $\operatorname{ad}_{B} x$.

LEMMA 2.10. Let $x=\sum \alpha_{i, j} p^{i}(p q)^{j}$ with $\alpha_{i, j_{0}} \neq 0$ for some $j_{0} \geq 2$ or $\alpha_{i_{0}, 1} \neq 0$ for some $i_{0} \geq 1$; then $F(x) \neq B$.

Proof. Since $B$ is a subalgebra of $A_{1}$ and $x \in B$, then $x$ is an element of $A_{1}$. Thus $x$ can be written as $x=\sum_{m, n} \beta_{m, n} p^{m} q^{n}$ where $m \geq n$. Let $r$ be the smallest nonnegative integer such that $\beta_{m, 0}=0$ for $m \geq r+1$ and $s$ be the smallest nonnegative integer such that $\beta_{0, n}=0$ for any $n \geq s+1$. Since $m \geq n$, then $\beta_{0, n}=0$ for any $n \geq 1$. Thus $s=0$.

Because of the conditions imposed on $x$, we have $\beta_{m_{1}, n_{1}} \neq 0$ for some $m_{1} \geq n_{1} \geq 1$. It is obvious that we can choose positive real numbers $\sigma, \rho$ with an irrational ratio such that

$$
\sigma m_{1}+\rho n_{1}>\rho s, \quad \sigma m_{1}+\rho n_{1}>r \sigma .
$$

Thus there exist $m_{2} \geq 0$ and $n_{2} \geq 0$ such that

$$
\beta_{m_{2}, n_{2}} \neq 0, \quad \sigma m_{2}+\rho n_{2}=v_{\sigma, \rho}(x) .
$$

Note that

$$
\sigma m_{2}+\rho n_{2}>\rho s, \quad \sigma m_{2}+\rho n_{2}>r \sigma .
$$

If $n_{2}=0$, then $m_{2}>r$. Thus $\beta_{m_{2}, n_{2}}=\beta_{m_{2}, 0}=0$, which is a contradiction to the definition of $r$. So $m_{2} \geq n_{2} \geq 1$. In addition, if $m_{2}=n_{2}=1$, then

$$
\sigma+\rho \leq \sigma m_{1}+\rho n_{1} \leq \sigma m_{2}+\rho n_{2}=\sigma+\rho .
$$

Thus $m_{1}=m_{2}$ and $n_{1}=n_{2}$, which is a contradiction. So $m_{2} \geq 2$ or $n_{2} \geq 2$.

By the second part of Lemma 2.8 about the associated polynomial of elements in $A_{1}$, we know that the $(\sigma, \rho)$-associated polynomial of $x$ is $\beta_{m_{2}, n_{2}} X^{m_{2}} Y^{n_{2}}$. Note that $m_{2} \leq n_{2}$. For $n=0,1,2, \ldots$, we set $y_{n}=(\operatorname{ad} x)^{n} p$. We will show by induction on $n$ that the $(\sigma, \rho)$-associated polynomial of $y_{n}$ is $\beta_{n} X^{n\left(m_{2}-1\right)+1} Y^{n\left(n_{2}-1\right)}$ with $\beta_{n} \in \mathbb{K}^{*}$. The statement is obviously true for $n=0$. Suppose that the statement is true for $n$. Note that

$$
m_{2}\left(n\left(m_{2}-1\right)\right)-n_{2}\left(n\left(m_{2}-1\right)+1\right)=-n_{2}+n n_{2}-n m_{2} \leq-n_{2}<0 .
$$

As a result of Lemma 2.9, the $(\sigma, \rho)$-associated polynomial of $y_{n+1}=\left[x, y_{n}\right]$ is given by

$$
\left(-n_{2}+n n_{2}-n m_{2}\right) \beta_{m_{2}, n_{2}} \beta_{n} X^{m_{2}+n\left(m_{2}-1\right)} Y^{n_{2}+n\left(n_{2}-1\right)-1} .
$$

Thus the statement is true for $n+1$. In addition, $v_{\sigma, \rho}\left(y_{n}\right)=\sigma\left(n\left(m_{2}-1\right)+1\right)+$ $\rho n\left(n_{2}-1\right)$. Since $m_{2} \geq 2$ or $n_{2} \geq 2$ and $m_{2} \geq n_{2} \geq 1$, then $v_{\sigma, \rho}\left(y_{n}\right)$ approaches $\infty$ as $n$ becomes large. Thus we have proved that $p \notin F(x)$. So $F(x) \neq B$. 
Theorem 2.11. If $F(x)=B$, then $x=\lambda p q+f(p)$ for some $\lambda \in \mathbb{K}$ and some polynomial $f(X) \in \mathbb{K}[X]$.

Proof. Let $x=\sum_{i, j} \alpha_{i, j} p^{i}(p q)^{j}$. Note that $F(x)=B$. By Lemma 2.10, $\alpha_{i, j}=0$ for all $j \geq 2$ and $\alpha_{i, 1}=0$ for all $i \geq 1$. Thus $x=\alpha_{0,1} p q+\sum_{i} \alpha_{i, 0} p^{i}$. We have proved the theorem.

2.3. The automorphism group $\operatorname{Aut}(B)$ of $B$. In this subsection we give a new determination of the automorphism group $\operatorname{Aut}(B)$ of $B$ using the results from the previous subsection.

LEMMA 2.12. If $x \in \mathbb{K}[p]$, then $N(x)=B$.

Proof. Since $x=f(p) \in \mathbb{K}[p]$, then $[x, p q]=[f(p), p q] \in \mathbb{K}[p]$. So $\left.\operatorname{~ad}_{B} x\right)^{2}$ $(p q)=0$. Thus $p q \in N(x)$. Since $p, p q \in N(x)$ and $B$ is generated by $p, p q$, then $N(x)=B$.

Proposition 2.13. We have $D(p q)=B$ and $C(p q)=\mathbb{K}[p q] . \quad$ In particular, $N(p q)=C(p q)=\mathbb{K}[p q]$.

Proof. Note that $[p q, p]=-p,[p q, p q]=0$ and $B$ is generated by $p, p q$; then $D(p q)=B$. It is easy to verify that $C(p q)=\mathbb{K}[p q]$. Thus

$$
C(p q)=D(p q) \cap N(p q)=B \cap N(p q)=N(p q)
$$

as desired.

LeMma 2.14. We have $N(p)=B, C(p)=\mathbb{K}[p]$. In particular, $D(p)=\mathbb{K}[p]$.

Proof. Since $[p, p q]=p$, then $\left(\operatorname{ad}_{B} p\right)^{2}(p q)=0$. So $p q \in N(p)$. Note that $B$ is generated by $p, p q$, thus $B=N(p)$. It is obvious that $C(p)=\mathbb{K}[p]$. Thus $D(p)=\mathbb{K}[p]$.

LEMMA 2.15. Let $\Phi$ be an automorphism of $B$, then $N(\Phi(x))=\Phi(N(x))$, $D(\Phi(x))=\Phi(D(x))$ and $F(\Phi(x))=\Phi(F(x))$.

PROOF. Since $\Phi$ is a $\mathbb{K}$-algebra automorphism, $\Phi(N(x)) \subset N(\Phi(x))$. Conversely, if $y \in N(\Phi(x))$, then $\operatorname{ad}_{B}(\Phi(x))^{n} y=0$ for sufficiently large $n$. So $\left(\operatorname{ad}_{B} x\right)^{n}\left(\Phi^{-1}(y)\right)=0$ for sufficiently large $n$. Thus $y \in \Phi(N(x))$. The rest of the proof is similar.

THEOREM 2.16. Let $\Phi$ be an automorphism of $B$; then $\Phi(p)=\lambda p, \lambda \in$ $\mathbb{K}^{*}, \Phi(p q)=p q+f(p)$ for some polynomial $f(X) \in \mathbb{K}[X]$.

PROOF. Let $\Phi$ be an automorphism of $B$; then $N(\Phi(p))=\Phi(N(p))=\Phi(B)=B$. So $F(\Phi(p))=B$. Thus, $\Phi(p)=f(p)+a p q$ by Theorem 2.11. Since $D(p q)=B$, then $(D(\Phi(p q)))=\Phi(B)=B$. Hence, $\Phi(p q)=b p q+g(p)$ by Theorem 2.11. If $a \neq 0$, then $p \notin N(f(p)+a p q)$; thus $N(\Phi(p)) \neq B$, which is a contradiction. Since $[p, p q]=p$ and $\Phi$ is an automorphism of $B$, then $[\Phi(p), \Phi(p q)]=\Phi(p)$. Therefore, 
$[f(p), b p q+g(p)]=[f(p), b p q]=f(p)$. Suppose that $f(p)=\sum f_{i} p^{i} ;$ then

$$
\sum b f_{i} i p^{i}=\sum f_{i} p^{i}
$$

So $f_{i}(b i-1)=0$. Now we need to show that $b=1$, which will imply that $f_{i}=0$ for $i \neq 1$. It is obvious that $f_{0}=0$. Now we must have $b=1 / i_{0}$ for some $i_{0} \geq 1$; otherwise $f=0$. Suppose that $i_{0}>1, \Phi(p)=f_{i_{0}} p^{i_{0}}$ where $i_{0} \geq 2$. This is impossible due to the fact that $\Phi(p)$ and $\Phi(p q)$ should generate $B$ as an algebra. So $b=1$ and $f_{i}=0$ for $i \neq 1$. Thus $\Phi(p)=\lambda p$ for some $\lambda \in \mathbb{K}^{*}$.

Corollary 2.17 (See $[1,17]$ ). We have $\operatorname{Aut}(B)=G$, where $G$ is the subgroup of Aut $(B)$ generated by the automorphisms $\Phi_{a, n, \lambda}$.

ProOf. Let $\Phi \in \operatorname{Aut}(B)$ be an automorphism of $B$. Then $\Phi(p)=a p$ for some $a \in K^{*}$ and $\Phi(p q)=p q+g(p)$ for some polynomial $g(X) \in \mathbb{K}[X]$. So $\Phi$ is a composition of automorphisms of the form $\Phi_{a, n, \lambda}$. Hence $\Phi \in G$. Thus $G=\operatorname{Aut}(B)$.

REMARK 2.18. This result was first obtained by Smith as a corollary of her main result in [17] by a different method. We can find a simpler determination which works for both zero and positive characteristics in [1].

\section{Strictly nilpotent elements and strictly semisimple elements in $B$}

In this section, we characterize all strictly nilpotent elements and strictly semisimple elements in $B$. As an application, we determine all mad associative subalgebras of $B$ and their framings.

3.1. Strictly nilpotent and strictly semisimple elements in $\boldsymbol{B}$. We are now ready to give a complete description of strictly nilpotent elements and strictly semisimple elements of $B$ in terms of the characterization given in the previous section and the automorphism group $\operatorname{Aut}(B)$.

First of all, we state the following important characterization of strictly nilpotent elements.

THEOREM 3.1. Let $x \in B-\mathbb{K}$ be a noncentral element. The following conditions are equivalent to each other:

(1) $x$ is strictly nilpotent;

(2) there exists an automorphism $\Phi$ of $B$ such that $\Phi(x) \in \mathbb{K}[p]$.

Furthermore, the following conditions are equivalent to each other:

(1) $x$ is strictly nilpotent and $C(x)=\mathbb{K}[x]$;

(2) there exists an automorphism $\Phi$ of $B$ such that $\Phi(x)=p+\beta$ for some $\beta \in \mathbb{K}$.

Proof. Suppose that $x$ is strictly nilpotent; then $N(x)=B$. Thus $F(x)=B$. Therefore, $x=f(p)+\lambda p q$ for some $f(X) \in \mathbb{K}[X]$ and $\lambda \in \mathbb{K}^{*}$. Since $N(x)=B$, then $\lambda=0$. So $x=f(p) \in \mathbb{K}[p]$. Let $\Phi \in \operatorname{Aut}(B)$ be any automorphism of $B$; then $\Phi(x)=\Phi(f(p)) \in \mathbb{K}[p]$. Conversely, if there exists an automorphism $\Phi \in \operatorname{Aut}(B)$ 
such that $\Phi(x) \in \mathbb{K}[p]$, then we know $x=f(p) \in \mathbb{K}[p]$ by the definition of the automorphism $\Phi$. So $N(x)=N(f(p))=B$. In addition, $C(x)=D(x) \cap N(x)=$ $D(x)$. The proof of the second equivalence is the same.

In particular, we have the following corollary.

COROLLARY 3.2. The set $\mathbb{K}[p]-\mathbb{K}$ is exactly the set of all strictly nilpotent elements of $B$.

PROOF. This follows from the structure of $\operatorname{Aut}(B)$.

Now we give a description of strictly semisimple elements of $B$.

THEOREM 3.3. An element $x$ is a strictly semisimple element of $B$ if and only if there exists an automorphism $\Phi \in \operatorname{Aut}(B)$ such that $\Phi(x)=\lambda p q$ for some $\lambda \in \mathbb{K}^{*}$.

Proof. Since $D(x)=B$ and $D(x) \subset F(x)$, then $B=F(x)=D(x)$. Thus $x=$ $\lambda p q+g(p)$. Supposing that $\lambda=0$, then $N(x)=B$; thus $C(x)=N(x) \cap D(x)=$ $N(x)=B$, which implies that $x \in \mathbb{K}$ due to the fact that the center of $B$ is reduced to $\mathbb{K}$. So $\lambda \neq 0$. Now we can choose a $\Phi \in G$ such that $\Phi(p q)=p q-1 / \lambda g(p)$. Thus $\Phi(x)=\lambda p q$. Conversely, if $\Phi(x)=\lambda p q$ for some $\lambda \neq 0 \in \mathbb{K}$ and some $\Phi \in G$, then $D(x)=\Phi^{-1}(D(\Phi(x)))=B$. Hence $x$ is strictly semisimple.

In particular, we have the following explicit description of elements in $\Delta_{3}$.

COROLlary 3.4. The following is true:

$$
\Delta_{3}=\left\{y \in B \mid y=\lambda p q+g(p), \text { where } \lambda \in \mathbb{K}^{*}, g(p) \in \mathbb{K}[p]\right\} .
$$

PROOF. This follows from Theorem 3.3 and the structure of the automorphism group $\operatorname{Aut}(B)$.

Furthermore, we have the following result concerning the eigenvalues of $\operatorname{ad}_{B} x$ for those elements $x \in \Delta_{3}$.

Corollary 3.5. Let $x \in B-\mathbb{K}$ be strictly semisimple; then there exists a $\rho \in \mathbb{K}$ such that the set of eigenvalues of $\operatorname{ad}_{B} x$ is $\mathbb{Z}_{\geq 0} \rho$.

Proof. Suppose $x$ is a strictly semisimple element of $B$. From the above theorem, there exists some $\Phi \in \operatorname{Aut}(B)$ such that $\Phi(x)=\lambda p q$ for some $\lambda \neq 0 \in \mathbb{K}$. Note that the set of eigenvalues for the inner derivation $\operatorname{ad}_{B}(\lambda p q)$ is the set $\mathbb{Z}_{\geq 0}(-\lambda)$. Therefore, the set of all eigenvalues of $\operatorname{ad}_{B} x$ is the set $\mathbb{Z}_{\geq 0}(-\lambda)$ as desired.

3.2. Maximal abelian ad-nilpotent associative subalgebras of $\boldsymbol{B}$. The mad associative subalgebras of $A_{1}$ were also determined by Dixmier in [9]. In [6], Berest and Wilson further determined the structure of the mad subalgebras of domains which are Morita equivalent to $A_{1}$. For more information about mad subalgebras and their applications, we refer the reader to [6] and the references therein. In this subsection, we determine the set of all mad subalgebras of $B$ and their framings. We will also study the action of $\operatorname{Aut}(B)$ on the set of framed mad subalgebras of $B$. 
Definition 3.6. A maximal abelian subalgebra $M$ of $B$ is called a mad subalgebra if every noncentral element of $M$ is strictly nilpotent.

It is easy to see that $\mathbb{K}[p]$ is a mad subalgebra of the algebra $B$. There are maximal abelian subalgebras of $B$ that are not mad subalgebras. For example, $\mathbb{K}[p q]$ is a maximal abelian subalgebra of $B$ that is not a mad subalgebra of $B$. Indeed, we will prove that $\mathbb{K}[p]$ is actually the only mad subalgebra of $B$.

First of all, we give the following description of mad subalgebras of $B$.

THEOREM 3.7. Every mad subalgebra $M$ of $B$ is of the form $M=\mathbb{K}[m]$ for some noncentral element $m$ in $B$. In particular, $M=\mathbb{K}[p]$.

PROOF. Since $M$ is a maximal abelian subalgebra of $B$, then by [9, Remark 4.9], we have $M=C(m)$ for some noncentral element $m \in B$. Since $m \in C(m)$ and $M=C(m)$ is a mad subalgebra of $B$, then $m$ is strictly nilpotent and $m \in \mathbb{K}[p]$. Thus $m=g(p)$ for some $g(p) \in \mathbb{K}[p]$. Therefore $M=C(m)=\mathbb{K}[p]$ as desired.

If $M$ is a mad subalgebra of $B$ such that $M=\mathbb{K}[m]$, then we shall call a choice of the generator $m$ for $M$ a framing of $M$, and the pair $(M, m)$ is called a framed mad subalgebra of $B$. Though the algebra $B$ has only one mad subalgebra $M=\mathbb{K}[p]$, the mad subalgebra $M$ has different framings other than $p$. However, it easy to see the following result.

LEMMA 3.8. The elements $m=\alpha p+\beta, \alpha \in \mathbb{K}^{*}, \beta \in \mathbb{K}$, are precisely the framings of the mad subalgebra $M=\mathbb{K}[p]$.

Let Mad B denote the set of all framed mad subalgebras of $B$. It is easy to see that the automorphism group $\operatorname{Aut}(B)$ is acting on the set Mad B. For any $\beta \in \mathbb{K}$, let Mad $\mathbf{B}_{\beta}$ denote the subset consisting of all framed mad subalgebras $(M, \alpha p+\beta)$ where $\alpha \in \mathbb{K}^{*}$. Then it is easy to see that $\operatorname{Aut}(B)$ acts transitively on $\mathbf{M a d} \mathbf{B}_{\beta}$. Let $\Gamma \subset \operatorname{Aut}(B)$ denote the subgroup of $\operatorname{Aut}(B)$ consisting of all automorphisms $\Phi \in \operatorname{Aut}(B)$ such that $\Phi(p q)=p q+g(p), \Phi(p)=p$, for some $g(p) \in \mathbb{K}[p]$.

THEOREM 3.9. We have the following:

$$
\operatorname{Mad} \mathbf{B}=\bigcup_{\beta \in K} \operatorname{Mad} \mathbf{B}_{\beta}
$$

and

$$
\operatorname{Aut}(B) / \Gamma \cong \operatorname{Mad} \mathbf{B}_{\beta}
$$

PROOF. It is obvious that Mad $\mathbf{B}=\bigcup_{\beta} \operatorname{Mad} \mathbf{B}_{\beta}$. The second equality follows from the fact that $\Gamma$ is the stabilizer of $(M, p+\beta)$ in $\operatorname{Aut}(B)$ and $\operatorname{Aut}(B)$ acts transitively on $\operatorname{Mad} \mathbf{B}_{\beta}$.

\section{Some useful results on elements in $\Delta_{i}, i=2,3,4,5$}

In this section, we investigate some useful properties of elements in $\Delta_{i}, i=2$, $3,4,5$. We first construct some specific elements to show that $\Delta_{2}, \Delta_{4}, \Delta_{5}$ are 
nonempty. In particular, for elements in $\Delta_{3}, \Delta_{4}, \Delta_{5}$, we derive some important results which will serve as a foundation for the study of Lie subalgebras of $B$.

4.1. Elements in $\Delta_{2}$ and $\Delta_{4}$. In this subsection we prove that $\Delta_{2}$ and $\Delta_{4}$ are nonempty by constructing concrete examples. Let $x \in B$; then $N(x)$ is a filtered subalgebra of $B$, which is filtered by $N(x, n), n \in \mathbb{Z}_{\geq 0}$. First of all, we have the following proposition.

Proposition 4.1. Let $x$ be an element of B. Noting that

$$
N(x, n)=\left\{b \in B \mid\left(\operatorname{ad}_{B} x\right)^{n+1}(b)=0\right\},
$$

then:

(1) the graded algebra $G=\bigoplus N(x, n+1) / N(x, n)$ associated with the filtered algebra $N(x)$ is a commutative domain;

(2) considered as a $C(x)=N(x, 0)$-module, each $N(x, n+1) / N(x, n)$ is of finite type.

Proof. Since $B$ is a subalgebra of $A_{1}$, then $x$ can be regarded as an element of $A_{1}$. By [9, Proposition 10.2], we know that

$$
G_{1}=\bigoplus N\left(x, A_{1}, n+1\right) / N\left(x, A_{1}, n\right)
$$

is a commutative domain. In addition, $G$ is a subalgebra of $G_{1}$. Thus it is a commutative domain. Using the same argument used in [9, Proof of Proposition 10.2], we can show that $N(x, n+1) / N(x, n)$ is an ideal of $C(x)$. Note that $C(x)$ is a commutative noetherian algebra. Thus $N(x, n+1) / N(x, n)$ is of finite type considered as a $C(x)$-module.

Proposition 4.2. Let $x, y$ be two nonscalar commuting elements of $B$. Then $N(x, n)=N(y, n)$ for $n \geq 0$; in particular, $N(x)=N(y)$.

Proof. Since $B$ is a subalgebra of $A_{1}$ and $N\left(x, A_{1}\right), N\left(x, A_{1}\right)$ are filtered algebras, then $N(x, B, n)=B \cap N\left(x, A_{1}, n\right), N(y, B, n)=B \cap N\left(y, A_{1}, n\right)$. Thus the proposition follows from [9, Proposition 10.3].

Corollary 4.3. Let $C$ be a maximal commutative subalgebra of $B$. If $C \cap \Delta_{1}$ is nonempty, then $C-\mathbb{K} \subset \Delta_{1}$. If $C \cap \Delta_{2}$ is nonempty, then $C-\mathbb{K} \subset \Delta_{2}$.

PROOF. If the intersection $C \cap \Delta_{1}$ is nonempty, then there exists an element $x \in$ $C \cap \Delta_{1}$ such that $N(x)=B$. Since $x, y$ commute with each other, then $N(y)=N(x)$ for any $b \in C-\mathbb{K}$. The second statement can be proved in the same way.

Proposition 4.4. Let $x \in B$, then the eigenvalues of $\operatorname{ad}_{B} x$ are linearly independent over $\mathbb{Q}$.

PROOF. The eigenvalues of $\operatorname{ad}_{B} x$ are also eigenvalues of $\operatorname{ad}_{A_{1}} x$. Thus the result follows from [9, Proposition 10.5]. 
Proposition 4.5. Let $x, y$ be two nonzero elements of $B$ such that $[x, y]=\lambda y$, where $\lambda \in \mathbb{K}^{*}$. Then:

(1) $x+y$ is a semisimple element;

(2) $y$ is a nilpotent element and $x^{i} y^{j} \in N(y)$ for any integers $i, j \geq 0$;

(3) if $C(x)=\mathbb{K}[x]$, then $\sum D(x,-\lambda r) \subset N(y)$.

PROOF. First of all, $[x+y, y]=\lambda y$. Thus $D(x+y) \neq C(x+y)$. By the classification, we know that $x+y$ is a semisimple element. Now $[y, x] \neq 0$ and $\left(\operatorname{ad}_{B} y\right)^{2} x=0$, thus $y$ is a nilpotent element and $x, y \in N(y)$. We have thus proved part (2). Now let us suppose that $C(x)=\mathbb{K}[x]$; then $C(x) \subset N(x)$. In addition, $\left(\operatorname{ad}_{B} y\right) D(x,-\lambda r) \subset D(x,-\lambda r+\lambda)$. Thus

$$
\left(\operatorname{ad}_{B} y\right)^{r} D(x,-\lambda r) \subset D(x, 0)=C(x) \subset N(y)
$$

for all $r \geq 0$, and we are done.

We now look at an example.

EXAMPLE 4.6. Let $a=-p q, b=p(p q)=p^{2} q$; then $[a, b]=b$. So $p^{2} q-p q$ is semisimple and $p^{2} q$ is nilpotent. In addition, $F\left(p^{2} q\right) \neq B$ and $F\left(p^{2} q-p q\right) \neq B$ by Lemma 2.10. So $b=p(p q)$ is in $\Delta_{2}$ and $a+b=p(p q)-p q$ is in $\Delta_{4}$.

We now state the following proposition.

PROPOSITION 4.7. The subsets $\Delta_{2}, \Delta_{4}$ of $B$ are nonempty.

4.2. Elements in $\Delta_{5}$. In this short subsection we show that $\Delta_{5}$ is also nonempty. To succeed, we only need to find some element $x \in B-\mathbb{K}$ such that $C(x)=N(x)=$ $D(x)$. In [9], Dixmier found a practical way to determine whether an element is in $\Delta_{5}$ or not; and he also gave a concrete example to show that $\Delta_{5}$ is nonempty. Since $B$ is a subalgebra of $A_{1}$, then $N(x, B)=B \cap N\left(x, A_{1}\right), C(x, B)=B \cap C\left(x, A_{1}\right)$. So it is enough to find some element in $\Delta_{5}\left(A_{1}\right) \cap B$, that will automatically be an element in $\Delta_{5}$.

First of all, we quote two results from [9] for the purposes of this subsection.

Lemma 4.8 [9, Lemma 7.3]. Let $\rho, \sigma \geq 1$ be integers. Let $x \in A_{1}, y \in F(x)$, and $v=v_{\rho, \sigma}(x), \omega=v_{\rho, \sigma}(y) ; f, g$ are $(\rho, \sigma)$-polynomials associated with $x, y$, respectively. We suppose that $v \geq \rho+\sigma+1$ and $f$ are not monomials. Then one of the following cases is true:

(1) $f^{\omega}$ is proportional to $g^{\nu}$;

(2) $\sigma \geq \rho+1, \sigma$ is a multiple of $\rho$ and $f(X, Y)$ is of the form $\lambda X^{\alpha}\left(X^{\sigma / \rho}+\mu Y\right)^{\beta}$ where $\lambda, \mu \in \mathbb{K}$ and $\alpha, \beta \geq 0$ are integers;

(3) $\rho \geq \sigma+1, \rho$ is a multiple of $\sigma$ and $f(X, Y)$ is of the form $\lambda Y^{\alpha}\left(Y^{\rho / \sigma}+\mu X\right)^{\beta}$ where $\lambda, \mu \in \mathbb{K}$ and $\alpha, \beta \geq 0$ are integers;

(4) $\rho=\sigma$, and $f(X, Y)$ is of the form $\lambda(\mu X+v Y)^{\alpha}\left(\mu^{\prime} X+v^{\prime} Y\right)^{\beta}$ where $\lambda, \mu, v, \mu^{\prime}, v^{\prime} \in \mathbb{K}$ and $\alpha, \beta \geq 0$ are integers. 
LeMma 4.9 [9, Proposition 7.4]. Let $\rho, \sigma \geq 1$ be integers, and $x \in A_{1}, v=v_{\rho, \sigma}(x)$, and $f$ be the $(\rho, \sigma)$-polynomial associated to $x$. Suppose that:

(1) $v \geq \rho+\sigma+1$;

(2) $f$ is not a monomial;

(3) we are not in cases (2)-(4) of the previous lemma.

Then $F(x)=C(x)$.

Here is another example.

EXAMPLE 4.10. Let

$$
x=p^{5}+(p q)^{2}=p^{5}+p^{2} q^{2}-p q ;
$$

then $v_{2,3}(x)=10$. So the $(2,3)$-associated polynomial of $x$ is $X^{5}+X^{2} Y^{2}$. By the previous lemmas, $F\left(x, A_{1}\right)=C\left(x, A_{1}\right)$. Thus $F(x, B)=C(x, B)$. Note that $x \in B$, so $x \in \Delta_{5}$.

As a result, we have the following proposition.

Proposition 4.11. The subset $\Delta_{5}$ of $B$ is nonempty.

4.3. Further results on elements in $\Delta_{\boldsymbol{i}}, \boldsymbol{i}=\mathbf{3}, \mathbf{4}, \mathbf{5}$. We now use the method of polarization to study elements in $\Delta_{i}, i=3,4,5$. Note that

$$
\operatorname{ad}(p q)\left(p^{n} f(p q)\right)=-n p^{n} f(p q)
$$

we call $n$ the weight of $p^{n} f(p q)$. So

$$
B=\bigoplus_{n \geq 0} p^{n} \mathbb{K}[p q]
$$

is a decomposition of $B$ with respect to the weight spaces of $\operatorname{ad}_{B}(p q)$. Hence $B$ is a $\mathbb{Z}_{\geq 0}$-graded algebra.

First of all, we state a reduction theorem for elements in $\Delta_{4}$.

THEOREM 4.12. Let $x \in \Delta_{4}$; then there exists an automorphism $\Phi \in \operatorname{Aut}(B)$ such that

$$
\Phi(x)=\alpha p q+\sum_{s \geq 1} p^{s} f_{s}(p q)
$$

where $\alpha \neq 0$ and $f_{S}(X)$ are polynomials in $X$.

Proof. Since $x \in \Delta_{4}$, then $D(x) \neq C(x)$. So we can choose an element $y \in D(x)$ such that $[x, y]=\lambda y$ where $\lambda \neq 0$. Let $x^{\prime}, y^{\prime}$ be the lowest weight components of $x, y$ with respect to the action of $\operatorname{ad}_{B}(p q)$. Then $x^{\prime} \in \mathbb{K}[p q]$ and $\left[x^{\prime}, y^{\prime}\right]=\lambda y^{\prime}$. By a direct calculation, $x^{\prime}=\alpha p q+\beta$ where $\alpha \neq 0$. If $\beta=0$, then we are done. Otherwise, we can choose a $\Phi \in \operatorname{Aut}(B)$ such that $\Phi(p q)=p q-\beta / \alpha$. Thus $\Phi\left(x^{\prime}\right)=\alpha p q$. We have thus shown that

$$
\Phi(x)=\alpha p q+\sum_{s \geq 1} p^{s} f_{s}(p q)
$$

for some $\Phi \in \operatorname{Aut}(B)$. 
THEOREM 4.13. Given any element $x \in \Delta_{3} \cup \Delta_{4}$, then $C(x)=\mathbb{K}[x]$.

PROOF. If $x \in \Delta_{3}$, the result is obvious. Suppose that $x \in \Delta_{4}$ and we choose an element $y \in C[x]$. Let $y^{\prime}$ be the lowest weight component of $\Phi(y)$, where $\Phi$ is chosen as in the proof of the former theorem for $x$. Since $y \in C(x)$, then $\Phi(y) \in C(\Phi(x))$. Hence $y^{\prime} \in \mathbb{K}[p q]$ by the reduction theorem. Let $y^{\prime}=f(p q)$ for some polynomial $f(X)$ and let us set $z=y-f(1 / \alpha x)$. Then $z \in C(x)$. Suppose that $z \neq 0$; then the lowest weight of $\Phi(z)$ is strictly positive. Since $[\Phi(z), \Phi(x)]=0$, the lowest weight of $\Phi(z)$ has to be zero. Thus we have a contradiction. So $z=0$. Hence $y=f(1 / \alpha x) \in \mathbb{K}[x]$ as desired.

THEOREM 4.14. Let $x \in \Delta_{4}$; then there exists a $\rho$ such that $\Lambda(x) \subset \mathbb{Z}_{\geq 0} \rho$.

PROOF. Let $x \in \Delta_{4}$; we choose an element $y \in D(x)$ such that $[x, y]=\lambda y$ where $\lambda \neq 0$. Let $l$ be the lowest weight of $\Phi(y)$ where $\Phi$ is chosen for $x$ as in the proof of the reduction theorem. Then $\lambda=-\alpha l$. Let us set $-\alpha=\rho$; then $\Lambda(x) \subset \mathbb{Z}_{\geq 0} \rho$. The proof is complete.

LEMMA 4.15. Given $y \in B$ such that $f(y) \in \Delta_{3}$ (respectively $f(y) \in \Delta_{4}$ ) where $f(X)$ is a polynomial, then $f(X)$ is linear polynomial and $y \in \Delta_{3}$ (respectively $\Delta_{4}$ ).

PROOF. Suppose that $y \in B$ and $f(y) \in \Delta_{3} \cup \Delta_{4}$ for some polynomial $f(X)$; then $C(f(y))=\mathbb{K}[f(y)]$. Since $y \in C(f(y))$, there exists a polynomial $g(X)$ such that $y=g(f(y))$. Since $B$ is a domain, $f, g$ are linear polynomials. We have proved the lemma.

LEMMA 4.16. Let $x \in B$. Then $x \in \Delta_{1}$ (respectively $\Delta_{2}$ ) if and only if $f(x) \in \Delta_{1}$ (respectively $\Delta_{2}$ ) where $f(X)$ is a nontrivial polynomial.

PROOF. The lemma follows directly from Corollary 4.3.

PROpOSITION 4.17. Let $x \in \Delta_{3} \cup \Delta_{4}$ and $f(X)$ be a polynomial such that $\operatorname{deg}(f)>1$; then $f(x) \in \Delta_{5}$.

PROOF. The proposition follows from the previous two lemmas.

Proposition 4.18. Given $x \in \Delta_{5}$, then $\mathbb{K}[x]-\mathbb{K} \subset \Delta_{5}$.

PROOF. The result follows directly from the previous two lemmas.

\section{Finite-dimensional Lie subalgebras of $B=\mathcal{U}(\mathfrak{r}(1))$}

In this section we determine all the finite-dimensional nonabelian Lie algebras that can be realized as Lie subalgebras of $B=\mathcal{U}(\mathfrak{r}(1))$, based on the results derived in the previous sections and the corresponding classification results for nonabelian Lie subalgebras of $A_{1}$ in [15]. From now on, we will assume that $\mathbb{K}=\mathbb{C}$. 
5.1. Finite-dimensional nonabelian Lie subalgebras that can be realized in $\boldsymbol{A}_{1}$. In this subsection we recall some results on the realizations of finite-dimensional nonabelian Lie algebras as Lie subalgebras of $A_{1}$ and the necessary notation from [15].

It is well known that the Lie algebras $\mathfrak{s l}(2), \mathfrak{s l}(2) \times \mathbb{C}$ and $\mathfrak{s l}(2) \ltimes \mathcal{H}_{3}$ (where $\mathcal{H}_{3}$ denotes the three-dimensional Heisenberg Lie algebra) can be realized as Lie subalgebras of the first Weyl algebra $A_{1}$. In [16], Simoni and Zaccaria proved that the only complex semisimple Lie algebra that can be realized in $A_{1}$ is $\mathfrak{s l}(2)$, and a remarkable property of realizations of $\mathfrak{s l}(2)$ in $A_{1}$ was proved by Joseph in [12], where he showed that the spectrum of the realization in $A_{1}$ of suitably normalized semisimple elements of $\mathfrak{s l}(2)$ is either $\mathbb{Z}$ or $2 \mathbb{Z}$. In [11], Igusa proved a necessary condition for two elements of $A_{1}$ to generate an infinite-dimensional Lie subalgebra.

In [15], Rausch de Traubenberg et al. determined all the finite-dimensional complex nonabelian Lie algebras that can be realized in $A_{1}$. Suppose that $\mathfrak{g}$ is a complex finite-dimensional nonabelian Lie algebra and let $A_{1}^{\mathfrak{g}}$ denote the set of all injective Lie algebra homomorphisms from $\mathfrak{g}$ to $A_{1}$. The following classification theorem was proved in [15] for $A=A_{1}$.

THEOREM 5.1 [15]. Let $\mathfrak{g}$ be a complex finite-dimensional nonabelian Lie algebra. Then $A_{1}^{\mathfrak{g}} \neq \emptyset$ if and only if $\mathfrak{g}$ is isomorphic to one of the following:

- $\quad \mathfrak{s l}(2), \mathfrak{s l}(2) \times \mathbb{C}, \mathfrak{s l}(2) \ltimes \mathcal{H}_{3}$;

- $\quad L_{n},(n \geq 2), \widetilde{L_{n}},(n \geq 2)$;

- $\quad \mathfrak{r}\left(i_{1}, \ldots, i_{n}\right)$ where $i_{1}<\cdots<i_{n}$ are positive integers.

Note that $L_{n}$ is a nilpotent, in fact filiform, Lie algebra, $\widetilde{L_{n}}$ is isomorphic to a semidirect product $\mathbb{C} \ltimes L_{n}$, and $\mathfrak{r}\left(i_{1}, \ldots, i_{n}\right)$ is isomorphic to a semidirect product $\mathbb{C} \ltimes \mathbb{C}^{n}$. Indeed, there are only a finite number of nonsolvable Lie algebras and only a discrete family of solvable Lie algebras that can be realized as Lie subalgebras of $A_{1}$. For more details, we refer the reader to [15].

Since $B=\mathcal{U}(\mathfrak{r}(1))$ is a subalgebra of $A_{1}$, a finite-dimensional complex Lie algebra can be realized in $A_{1}$ as long as it can be realized in $B$. Thus a finite-dimensional nonabelian Lie subalgebra of $B$ is among the Lie algebras listed in the previous theorem. To further determine the finite-dimensional complex nonabelian Lie algebras that can be realized in $B$, it suffices to determine which algebras listed in the previous theorem can indeed be realized in $B$. This can be done thanks to the results obtained in the previous sections. In particular, we will prove that the only finite-dimensional complex nonabelian Lie algebras that can be realized in $B$ are of the type $\mathfrak{r}\left(i_{1}, \ldots, i_{n}\right)$.

\subsection{A characterization of $\Delta_{1} \cup \Delta_{3}$ in terms of exponentiation.}

Definition 5.2 (See [15, Definition 2.4]). Let $z \in B$. One says that $\operatorname{ad}_{B}(z)$ can be exponentiated if there exists a group homomorphism $\phi: \mathbb{C} \longrightarrow \operatorname{Aut}(B)$ such that:

(1) for all $a \in B$, the vector space $V_{a}=\langle\phi(t)(a): t \in \mathbb{C}\rangle$ is finite-dimensional;

(2) $\phi_{a}: \mathbb{C} \longrightarrow V_{a}$ is holomorphic and $d /\left.(d t)\right|_{0} \phi_{a}(t)=[z, a]$ where $\phi_{a}(t)=$ $\phi(t)(a)$. 
EXAMPLE 5.3. If $z \in B$ is such that $\operatorname{ad}_{B}(z)$ is locally nilpotent, then $\operatorname{ad}_{B}(z)$ can be exponentiated in this sense. If $z=\lambda p q$ for some $\lambda \in \mathbb{C}^{*}$, then $\operatorname{ad}_{B}(z)$ can be also exponentiated by the group homomorphism

$$
\phi: \mathbb{C} \longrightarrow \operatorname{Aut}(B)
$$

which is given on the canonical basis $\left\{(p q)^{i} p^{j} \mid i, j \in \mathbb{Z}_{\geq 0}\right\}$ by

$$
\phi(t)\left((p q)^{i} p^{j}\right)=e^{\lambda t(-j)}(p q)^{i} p^{j} .
$$

As in [15], we have the following lemma.

Lemma 5.4. Suppose that $\operatorname{ad}_{B} z$ can be exponentiated in the above sense. Then:

(1) for all $a \in B$, the vector space $V_{a}$ is stable under the action of $\operatorname{ad}_{B} z$;

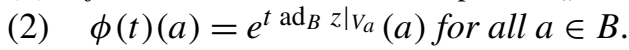

We now have the following characterization of elements in $\Delta_{1} \cup \Delta_{3}$ in terms of exponentiation.

Proposition 5.5. Let $z \in B$ be noncentral. Then $\operatorname{ad}_{B} z$ can be exponentiated if and only if $z \in \Delta_{1} \cup \Delta_{3}$.

PROOF. The proof is analogous to that for $A_{1}$ in [15]. Suppose that $z \notin \Delta_{1} \cup \Delta_{3}$. Recall that

$$
F(z)=\left\{b \in B \mid \operatorname{dim}\left(\left\langle\operatorname{ad}^{n}(z)(b), n \in \mathbb{N}\right\rangle\right)<\infty\right\}
$$

and $F(z)=D(z) \cup N(z)$. Since $z \notin \Delta_{1} \cup \Delta_{3}$, then $F(z) \neq B$. Let $b \in B-F(z)$. Then by the hypothesis, $V_{b}$ is a finite-dimensional vector space containing $b$ and stable under the inner derivation $\operatorname{ad}_{B}(z)$. Thus $\left\langle\operatorname{ad}_{B}^{n}(z)(b) \mid n \in \mathbb{N}\right\rangle \subseteq V_{b}$ is also finitedimensional, which is a contradiction.

Conversely, suppose that $z \in \Delta_{1} \cup \Delta_{3}$. Then by Theorems 3.1 and 3.3, up to an automorphism of $B, z$ is equal to $\lambda p q$ for some $\lambda \in \mathbb{C}^{*}$ or to a polynomial $g(p)$ in $p$. $\operatorname{Both}_{\operatorname{ad}_{B}}(\lambda p q)$ and $\operatorname{ad}_{B}(g(p))$ can be exponentiated. Thus $\operatorname{ad}_{B} z$ can be exponentiated.

5.3. Examples of Lie subalgebras of $\boldsymbol{B}=\mathcal{U}(\mathfrak{r}(\mathbf{1}))$. In this subsection we will construct some examples of both infinite- and finite-dimensional Lie subalgebras of $B$. Though we are particularly interested in finite-dimensional Lie nonabelian subalgebras of $B$, we will also discuss some examples of infinite-dimensional abelian subalgebras of $B$.

Since all maximal abelian Lie subalgebras of $B$ (which is regarded as a Lie algebra) are precisely maximal abelian associative subalgebras of $B$ (which is regarded as an associative algebra), we can state the following result.

PROPOSITION 5.6. Any maximal abelian Lie subalgebra of $B$ is the centralizer $C(b)$ of some noncentral element $b$ in $B$. 
PRoOF. This follows from the fact that maximal abelian Lie subalgebras of $B$ are precisely maximal abelian associative subalgebras of $B$. By [9, Remark 4.9], we know that all maximal abelian associative subalgebras of $B$ are precisely centralizers of noncentral elements of $B$. Thus we are done.

EXAmple 5.7. The set $\left\{p^{i} \mid i \geq 0\right\}$ spans a maximal abelian Lie subalgebra of $B$, which is infinite-dimensional.

EXAMPLE 5.8. The set $\left\{(p q)^{i} \mid i \geq 0\right\}$ spans a maximal abelian Lie subalgebra of $B$, which is infinite-dimensional.

We now look at some examples of finite-dimensional nonabelian Lie subalgebras of $B$.

EXAMPLE 5.9. The set $\{p q, p\}$ spans a nonnilpotent solvable Lie subalgebra of $B$. Indeed, the Lie subalgebra spanned by $\{p q, p\}$ is isomorphic to the Lie algebra $\mathfrak{r}(1)$.

EXAMPLE 5.10. Let $i_{k}, k=1, \ldots, n$, be distinct nonnegative integers which are not all zero. Then the set $\left\{p q, p^{i_{1}}, \ldots, p^{i_{n}}\right\}$, for $n \geq 2$, spans a nonnilpotent solvable Lie subalgebra $\mathfrak{r}\left(i_{1}, \ldots, i_{n}\right)$ of $B$. The derived algebra of $\mathfrak{r}\left(i_{1}, \ldots, i_{n}\right)$ is $n$-dimensional and abelian. Let $X_{0}=p q, X_{k}=p^{i_{k}}$ for $k=1, \ldots, n$; then the only nonabelian relations are

$$
\left[X_{0}, X_{k}\right]=-i_{k} X_{k}
$$

It is easy to see that

$$
\mathfrak{r}\left(i_{1}, \ldots, i_{n}\right) \equiv \mathfrak{r}\left(m i_{1}, \ldots, m i_{n}\right),
$$

for any $m \geq 1$, and

$$
\mathfrak{r}\left(i_{1}, \ldots, i_{n}\right) \equiv \mathfrak{r}\left(i_{\sigma(1)}, \ldots, i_{\sigma(n)}\right),
$$

for any permutation $\sigma \in S_{n}$.

5.4. Finite-dimensional Lie algebras that can be realized in $B=\mathcal{U}(\mathfrak{r}(1))$. In this subsection we prove that $\mathfrak{r}\left(i_{1}, \ldots, i_{n}\right)$ are the only finite-dimensional nonabelian Lie algebras that can be realized as Lie subalgebras of $B$.

First of all, we need the following definition for realizations of Lie algebras in $B$.

Definition 5.11. Let $\mathfrak{g}$ be a complex Lie algebra. Let us set

$$
\begin{gathered}
B^{\mathfrak{g}}=\{r \in \operatorname{Hom}(\mathfrak{g}, B) \mid \mathrm{r} \text { is an injective linear map such that } \\
r([a, b])=r(a) r(b)-r(b) r(a) \text { for } a, b \in B\} .
\end{gathered}
$$

If $B^{\mathfrak{g}} \neq \emptyset$, then we will say that the Lie algebra $\mathfrak{g}$ can be realized as a Lie subalgebra of $B$ and an element of $B^{\mathfrak{g}}$ will be called a realization of $\mathfrak{g}$ in $B$.

LEMMA 5.12. The Lie algebras $\mathfrak{s l}(2), \mathfrak{s l}(2) \times \mathbb{C}$ and $\mathfrak{s l}(2) \ltimes \mathcal{H}_{3}$ cannot be realized as Lie subalgebras of $B$. 
PROOF. Since all these Lie algebras have a Lie subalgebra which is isomorphic to $\mathfrak{s l}(2)$, it suffices to prove that $\mathfrak{s l}(2)$ cannot be realized as a Lie subalgebra of $B$. Suppose that $r: \mathfrak{s l}(2) \longrightarrow B$ is a realization of $\mathfrak{s l}(2)$ as a Lie subalgebra of $B$; and let $\left\{e_{0}, e_{1}, e_{2}\right\}$ be a standard basis of $\mathfrak{s l}(2)$ with $e_{0}$ denoting the semisimple element. Then $r\left(e_{0}\right)$ is a semisimple element of $B$. Note that the set of the eigenvalues of $\operatorname{ad}_{B} r\left(e_{0}\right)$ contains the set $2 \mathbb{Z}$ as a subset, which is a contradiction to Corollary 3.5 and Theorem 4.14. Thus $\mathfrak{s l}(2)$ cannot be realized as a Lie subalgebra of $B$. We have proved the lemma.

LEMMA 5.13. The Lie algebras $L_{n}$ and $\widetilde{L_{n}}$ cannot be realized as Lie subalgebras of $B$.

PROOF. Since these Lie algebras have a Lie subalgebra isomorphic to $L_{n}$, it suffices to prove that $L_{n}$ cannot be realized as a Lie subalgebra of $B$. Suppose that $r: L_{n} \longrightarrow B$ is a realization of $L_{n}$ in $B$. By [15, Lemma 4.14], there exist elements $a, b \in L_{n}$ such that $[r(a), r(b)]=1$, which is impossible in $B$. Thus we have proved that $L_{n}$ cannot be realized in $B$ as a Lie subalgebra.

THEOREM 5.14. The Lie algebras $\mathfrak{r}\left(i_{1}, \ldots, i_{n}\right)$ are the only finite-dimensional nonabelian Lie algebras that can be realized as Lie subalgebras of $B$.

PROOF. It is easy to see that $\mathfrak{r}\left(i_{1}, \ldots, i_{n}\right)$ can be realized as Lie subalgebras of $B$. Thus by Theorem 5.1 and Lemmas 5.12 and $5.13, \mathfrak{r}\left(i_{1}, \ldots, i_{n}\right)$ are the only finitedimensional nonabelian Lie algebras that can be realized as Lie subalgebras of $B$.

\section{Realizations of the Lie algebra $\mathfrak{r}(1)$ in $B$}

Let $u, v$ denote the basis elements of the Lie algebra $\mathfrak{r}(1)$ which satisfy the commutator relation $[u, v]=v$. It is obvious that $\mathfrak{r}(1)$ can be realized as a Lie subalgebra of $B$ by sending $u, v$ to $-p q, p$, respectively. In this section we study the family of all realizations of the Lie algebra $\mathfrak{r}(1)$ in $B$. Recall that $B^{\mathfrak{r}(1)}$ denotes the set of all realizations of the Lie algebra $\mathfrak{r}(1)$ in $B$.

6.1. A partition of $\boldsymbol{B}^{\mathfrak{r}(\mathbf{1})}$. In this subsection we prove some results on the realizations of $\mathfrak{r}(1)$ in $B$, which will be used to partition $B^{\mathfrak{r}(1)}$ into a disjoint union of two sets.

Let $r \in B^{\mathfrak{r}(1)}$ be a realization of $\mathfrak{r}(1)$ in $B$; then we have the following lemma.

LEMMA 6.1. The element $r(u)$ is in $\Delta_{3} \cup \Delta_{4}$ and the element $r(v)$ is in $\Delta_{1} \cup \Delta_{2}$.

PROOF. Since $u$ is semisimple considered as an element of the Lie algebra $\mathfrak{r}(1)$, then there exists an element $z \in \mathfrak{r}(1)$ such that $[u, z]=\lambda z$ for some $\lambda \in \mathbb{C}^{*}$. Thus $r(z) \in D(r(u))$ and $r(z) \notin C(r(u))$. Therefore, $C(r(u)) \neq D(r(u))$, which implies $r(u) \in \Delta_{3} \cup \Delta_{4}$. Similarly, we can prove that $r(v) \in \Delta_{1} \cup \Delta_{2}$. 
THEOREM 6.2. Let $r \in B^{\mathfrak{r}(1)}$ be a realization of $\mathfrak{r}(1)$ in $B$. Then there exists an automorphism $\Phi \in \operatorname{Aut}(B)$ such that

$$
\Phi(r(u))=\frac{-1}{n} p q+\sum_{s \geq 1} p^{s} g_{s}(p q)
$$

and

$$
\Phi(r(v))=\sum_{j \geq n} p^{j} h_{j}(p q)
$$

for some $n \in \mathbb{N}$ and $g_{s}(p q), h_{j}(p q) \in \mathbb{C}[p q]$.

Proof. By Lemma 6.1, $r(u) \in \Delta_{3} \cup \Delta_{4}$. Thus by Theorems 3.3 and 4.12, there exists a $\Phi \in \operatorname{Aut}(B)$ such that

$$
\Phi(r(u))=\lambda p q+\sum_{s \geq 1} p^{s} g_{s}(p q)
$$

for some $\lambda \neq 0$. Since $[u, v]=v$, then $[r(u), r(v)]=r(v)$. Since $\Phi$ is an automorphism of $B$, then $[\Phi(r(u)), \Phi(r(v))]=\Phi(r(v))$. By a direct calculation, $\lambda=-1 / n$ and $\Phi(r(v))=\sum_{j \geq n} p^{j} h_{j}(p q)$. Thus we have proved the theorem.

The following result is obvious.

COROLlary 6.3. If $r(v)$ is strictly nilpotent in $B$, then $r(u)$ is strictly semisimple in $B$.

Let us set

$$
B_{1}^{\mathfrak{r}(1)}=\left\{r \in B^{\mathfrak{r}(1)} \mid r(v) \in \Delta_{1}\right\}
$$

and

$$
B_{2}^{\mathfrak{r}(1)}=\left\{r \in B^{\mathfrak{r}(1)} \mid r(v) \in \Delta_{2}\right\} .
$$

Then it is easy to see that we have the following partition of $B^{\mathfrak{r}(1)}$.

Proposition 6.4. We have $B^{\mathfrak{r}(1)}=B_{1}^{\mathfrak{r}(1)} \cup B_{2}^{\mathfrak{r}(1)}$.

PROOF. This follows directly from Lemma 6.1.

Lemma 6.5. Let $a \in B, \mu \in \mathbb{C}^{*}$ be such that $[a, p]=-\mu p$. Then there exists $\Phi \in$ $\operatorname{Aut}(B)$ such that $\Phi(a)=\mu p q$.

Proof. Note that $[a, p]=-\mu p$ if and only if $[a-\mu p q, p]=0$ if and only if $a-\mu p q \in C(p)$. Since $C(p)=\mathbb{C}[p]$, then $a=\mu p q+f(p)$ for some $f(p) \in \mathbb{C}[p]$. Thus there exists an automorphism $\Phi \in \operatorname{Aut}(B)$ such that $\Phi(a)=\mu p q$.

LEMMA 6.6. Let $a \in B, \lambda \in \mathbb{C}^{*}$ and let $g(p)=\sum_{k=0}^{n} b_{k} p^{k}$ be a polynomial in $p$ of degree $n$. If $[a, g(p)]=\lambda g(p)$, then there exists an automorphism $\Phi \in \operatorname{Aut}(B)$ such that $[\Phi(a), p]=(\lambda / n) p$.

Proof. Since $[a, g(p)]=\lambda g(p)$ for some $\lambda \neq 0$, then $C(a) \neq D(a)$. By Theorem 2.6, we know that $a$ is semisimple (in $\Delta_{3} \cup \Delta_{4}$ ). By Theorems 3.3 and 4.12, 
we know that there exists an automorphism $\Phi \in \operatorname{Aut}(B)$ such that

$$
\Phi(a)=\beta p q+\sum_{i \geq 1} p^{i} f_{i}(p q)
$$

for some $\beta \in \mathbb{C}^{*}$. By direct calculations, we further know that we can choose $\Phi$ such that $\Phi(a)=(-\lambda / n) p q$. Thus we are done.

Proposition 6.7. Let $r \in B^{\mathfrak{r}(1)}$ be a realization of $\mathfrak{r}(1)$ in $B$. Then $r \in B_{1}^{\mathfrak{r}(1)}$ if and only if $\Phi(r(u))=(-1 / n) p q$ and $\Phi(r(v))=p^{n}$ for some $n \in \mathbb{N}, \Phi \in \operatorname{Aut}(B)$.

PROOF. This follows from the previous two lemmas.

Furthermore, we have the following corollary.

COROLlary 6.8. Let $r \in B^{\mathfrak{r}(1)}$ be a realization of $\mathfrak{r}(1)$ in $B$. Then $r \in B_{1}^{\mathfrak{r}(1)}$ if and only if $r(u)=(-1 / n) p q+g(p)$ and $r(v)=\lambda p^{n}$ for some $n \in \mathbb{N}, \lambda \in \mathbb{C}^{*}, g(p) \in$ $\mathbb{C}[p]$.

6.2. A family $\mathcal{N}$ of realizations of $\mathfrak{r}(\mathbf{1})$ in $\boldsymbol{B}$. In this subsection, we study the orbits of a particular family $\mathcal{N}$ of realizations of $\mathfrak{r}(1)$ in $B$ under the action of $\operatorname{Aut}(B)$. Indeed, we will prove that $B_{1}^{\mathfrak{r}(1)}$ is the union of these orbits.

Let us set

$$
\mathcal{N}=\left\{r_{n} \mid n \in \mathbb{N}\right\}
$$

where $r_{n}$ are realizations of $\mathfrak{r}(1)$ in $B$, which are defined by $r_{n}(u)=$ $(-1 / n) p q, r_{n}(v)=p^{n}$.

It is easy to see that the automorphism group $\operatorname{Aut}(B)$ is acting on the set $B^{\mathfrak{r}(1)}$ and we have the following proposition.

PROpOSITION 6.9. The elements in $\mathcal{N}$ are not equivalent to each other under the action of $\operatorname{Aut}(B)$.

PROOF. This is obvious and we omit the details.

DEFINITION 6.10. We fix the following notation:

- $\mathcal{D}=\left\{r \in B^{\mathfrak{r}(1)} \mid\right.$ there exists $\Phi \in \operatorname{Aut}(B)$ such that $\left.\Phi(r) \in \mathcal{N}\right\}$;

- $\mathcal{D}_{1}=\left\{r \in B^{\mathfrak{r}(1)} \mid\right.$ there exists $z \in \mathfrak{r}(1)-\{0\}$ such that $\left.r(z) \in \Delta_{1}\right\}$;

- $\quad \mathcal{D}_{1}^{\prime}=\left\{r \in B^{\mathfrak{r}(1)} \mid\right.$ there exists $z \in \mathfrak{r}(1)-\{0\}$ such that $[r(z), b]=0$

for some $\left.b \in \Delta_{1}\right\}$;

- $\mathcal{E}=\left\{r \in B^{\mathfrak{r}(1)} \mid\right.$ there exists a nilpotent element $z \in \mathfrak{r}(1)-\{0\}$

such that $\operatorname{ad}_{B} r(z)$ can be exponentiated\}.

We have the following equivalent descriptions of $B_{1}^{\mathfrak{r}(1)}$.

THEOREM 6.11. We have

$$
B_{1}^{\mathfrak{r}(1)}=\mathcal{D}=\mathcal{D}_{1}=\mathcal{D}_{1}^{\prime}=\mathcal{E}
$$


PRoOF. If $r \in B_{1}^{\mathfrak{r}(1)}$, then $r(v)=\mu p^{n}$ and $r(u)=(-1 / n) p q+g(p)$ for some $n \in$ $\mathbb{N}, \mu \in \mathbb{C}^{*}, g(p) \in \mathbb{C}[p]$ by Corollary 6.8. It is easy to see that there exists an automorphism $\Phi \in \operatorname{Aut}(B)$ such that $\Phi(r) \in \mathcal{N}$. Thus $r \in \mathcal{D}$, which implies that $B_{1}^{\mathfrak{r}(1)} \subset \mathcal{D}$.

If $r \in \mathcal{D}$, then there exists an automorphism $\Phi \in \operatorname{Aut}(B)$ such that $\Phi(r) \in \mathcal{N}$. In particular, $\Phi(r)(u)=(-1 / n) p q$ and $\Phi(r)(v)=p^{n}$ for some $n \in \mathbb{N}$. So $r(v)=\lambda p^{n}$ for some $\lambda \in \mathbb{C}^{*}$. Thus $r(v) \in \Delta_{1}$, which implies that $r \in \mathcal{D}_{1}$. So $\mathcal{D} \subseteq \mathcal{D}_{1}$.

It is obvious that $\mathcal{D}_{1} \subseteq \mathcal{D}_{1}^{\prime}$. Now we prove that $\mathcal{D}_{1}^{\prime} \subseteq \mathcal{E}$. Let $r \in \mathcal{D}_{1}^{\prime}$; then there exists $z \in \mathfrak{r}(1)-\{0\}$ such that $[r(z), b]=0$ for some $b \in \Delta_{1}$. Thus $r(z) \in C(b)=\Delta_{1} \cup \mathbb{C}$. However, $r(z)$ is not in $\mathbb{C}$, which implies that $z=\alpha v$ for some $\alpha \in \mathbb{C}^{*}$. Thus $r \in \mathcal{E}$, which implies that $\mathcal{D}_{1}^{\prime} \subseteq \mathcal{E}$.

If $r \in \mathcal{E}$, there exists a nilpotent element $z \in-\{0\}$ such that $\operatorname{ad}_{B} r(z)$ can be exponentiated. Since $z$ is nilpotent in $\mathfrak{r}(1)$, then $r(z) \in \Delta_{1} \cup \Delta_{2}$. Since $r(z)$ can be exponentiated, then $r(z) \in \Delta_{1}$. Once again, since $z$ is nilpotent in the Lie algebra $\mathfrak{r}(1)$, then we must have $z=\alpha v$ for some $\alpha \in \mathbb{C}^{*}$. Thus $r(v) \in \Delta_{1}$, which implies that $r \in B_{1}^{\mathfrak{r}(1)}$. The proof is thus complete.

It is easy to see the following corollary.

COROLlary 6.12. Let $r \in B^{\mathfrak{r}(1)}$ be a realization of $\mathfrak{r}(1)$ in $B$. The following are equivalent:

(1) $\quad r(\mathfrak{r}(1)-\{0\}) \subseteq \Delta_{1} \cup \Delta_{3}$;

(2) for all $z \in \mathfrak{r}(1)-\{0\}, r(z)$ can be exponentiated;

(3) there exists an automorphism $\Phi \in \operatorname{Aut}(B)$ such that $\Phi(r)=r_{n}$ for some $n \in \mathbb{N}$.

6.3. Other realizations of $\mathfrak{r}(\mathbf{1})$ in $\boldsymbol{B}$. In this subsection, we present some examples of realizations of $\mathfrak{r}(1)$ in $B$ that are not in $B_{1}^{\mathfrak{r}(1)}$. Unfortunately, the question of explicitly determine all elements in $B_{2}^{\mathfrak{r}(1)}$ remains open.

Let us set

$$
\begin{gathered}
\mathcal{M}=\left\{r_{n, h} \in B^{\mathfrak{r}(1)} \mid r_{n, h}(u)=\frac{-1}{n} p q, r_{n, h}(v)=p^{n} h(p q),\right. \\
\text { for some } h(p q) \in \mathbb{C}[p q]-\mathbb{C}\} .
\end{gathered}
$$

Then it is obvious that no element of $\mathcal{M}$ is in the set $B_{1}^{\mathfrak{r}(1)}$ and no two elements of $\mathcal{M}$ are equivalent under the action of $\operatorname{Aut}(B)$.

Definition 6.13. Let us fix the following notation:

- $\quad \mathcal{D}^{\prime}=\left\{r \in B^{\mathfrak{r}(1)} \mid\right.$ there exists $\Phi \in \operatorname{Aut}(B)$ such that $\left.\Phi(r) \in \mathcal{M}\right\}$;

- $\mathcal{D}_{2}=\left\{r \in B^{\mathfrak{r}(1)} \mid\right.$ there exists $z \in \mathfrak{r}(1)-\{0\}$ such that $\left.r(z) \in \Delta_{2}\right\}$.

It is easy to prove the following result.

Proposition 6.14. We have $\mathcal{D}^{\prime} \subseteq \mathcal{D}_{2} \subseteq B_{2}^{\mathfrak{r}(1)}$. 
6.4. The isotropy groups. Recall that if a group $\mathrm{G}$ acts on a set $X$, the isotropy of $x \in X$ in $G$ is by definition the subgroup $\{g \in G \mid g \cdot x=x\}$. In this subsection we calculate the isotropy group of the elements $f_{n}, f_{n, h}$ under the action of the group $\operatorname{Aut}(B)$. It is easy to see the following.

PROPOSITION 6.15. The isotropy groups of $r_{n}$ and $r_{n, h}$ are trivial.

REMARK 6.16. It might be useful to consider the orbits of elements in $B_{2}^{\mathfrak{r}(1)}$ under the action of the group $\operatorname{Aut}(B) \times \operatorname{Aut}_{\text {Lie }}(\mathfrak{r}(1))$ where $\operatorname{Aut}_{\text {Lie }}(\mathfrak{r}(1))$ denotes the Lie algebra automorphism group of the Lie algebra $\mathfrak{r}(1)$.

\section{References}

[1] J. Alev and F. Dumas, 'Invariants du corps de Weyl sous l'action de groupes finis (in French) [Invariants of the Weyl field under the action of finite groups]', Comm. Algebra 25 (1997), $1655-1672$.

[2] V. V. Bavula, 'Dixmier's problem 5 for the Weyl algebra', J. Algebra 283 (2005), 604-621.

[3] V. V. Bavula, 'Dixmier's problem 6 for somewhat commutative algebras and Dixmier's problem 3 for the ring of differential operators on a smooth irreducible affine curve', J. Algebra Appl. 4 (2005), 577-586.

[4] V. V. Bavula, 'Dixmier's problem 6 for the Weyl algebra (the generic type problem)', Comm. Algebra 34 (2006), 1381-1406.

[5] A. Berlinson and J. Bernstein, 'Localisation de g-modules', C. R. Acad. Sci. Paris 292 (1981), 15-18.

[6] Y. Berest and G. Wilson, 'Mad subalgebras of rings of differential operators on curves', Adv. Math. 212 (2007), 163-190.

[7] I. N. Bernšter̆n, 'Modules over a ring of differential operators. Study of the fundamental solutions of equations with constant coefficients', Funct. Anal. Appl. 5 (1971), 89-101.

[8] I. N. Bernšteĭn, 'The analytic continuation of generalized functions with respect to a parameter', Funct. Anal. Appl. 6 (1972), 273-285.

[9] J. Dixmier, 'Sur les algèbres de Weyl', Bull. Soc. Math. France 96 (1968), 209-242.

[10] J. Dixmier, 'Sur les algèbres de Weyl. II', Bull. Sci. Math. (2) 94 (1970), 289-301.

[11] J. Igusa, 'On Lie algebras generated by two differential operators', Progr. Math. 14 (1981), $187-195$.

[12] A. Joseph, 'A characterization theorem for realizations of sl(2)', Proc. Cambridge Philos. Soc. 75 (1974), 119-131.

[13] A. Joseph, 'The Weyl algebra—semisimple and nilpotent elements', Amer. J. Math. 97 (1975), $597-615$.

[14] M. Kashiwara, ' $B$-functions and holonomic systems. Rationality of roots of $B$-functions', Invent. Math. 38 (1976), 33-53.

[15] M. Rausch de Traubenberg, M. Slupinski and A. Tanasa, 'Finite-dimensional Lie subalgebras of the Weyl algebra', J. Lie Theory 16 (2006), 427-454.

[16] A. Simoni and F. Zaccaria, 'On the realization of semi-simple Lie algebras with quantum canonical variables', Nuovo Cimento A (10) 59 (1969), 280-292.

[17] M. K. Smith, 'Automorphisms of enveloping algebras', Comm. Algebra 11 (1983), 1769-1802.

[18] H. Weyl, The Theory of Groups and Quantum Mechanics (Dover, New York, 1950).

XIN TANG, Department of Mathematics \& Computer Science, Fayetteville State University, Fayetteville, NC 28301, USA

e-mail: xtang@uncfsu.edu 